\title{
EDITORIAL
}

\section{What progress looks like in NEC research}

\section{Journal of Perinatology (2011) 31, 149; doi:10.1038/jp.2010.164}

We are finally seeing the emergence of clinical insight into one of the most recalcitrant and entrenched fields of neonatal research: necrotizing enterocolitis (NEC) is beginning to unravel into its composite etiologies. In this edition of The Journal, we include two new articles examining the association between NEC and packed red blood cell transfusions. The number of publications on this topic has blossomed, both in our journal and others, resulting in an accelerated investigation of this relationship compared with past literature on NEC-related topics. Much of this has to do with the ubiquity of neonatal databases and the evolution of group think through electronic media. However, equally essential to this advance is a fundamental conceptual change in how we think about NEC. In decades past, NEC was an umbrella diagnosis under which all forms must somehow fit. Today, there is an emerging consensus that NEC is an end-stage condition that infants can acquire through different routes. ${ }^{1}$

It is through this reductionist approach that there is renewed hope for the future. Basic science has taught us much about the underlying susceptibilities and mechanisms of NEC, but it has not yielded a magic bullet. Although still in its infancy, NEC reductionism is offering fresh ideas for prevention and treatment through delineation and identification of distinct high-risk subgroups. ${ }^{1}$ Transfusion-associated NEC is one of the newest and most promising subgroups for intervention thus far.

In a seminal manuscript published on this topic earlier this year, the authors from the Intermountain Health System showed that transfusion-associated NEC occurs relatively late in the hospital course of predominately very low birth weight infants. ${ }^{2}$ In this edition, Singh et al. demonstrate that the underlying extent of anemia is a confounding risk factor, independent of transfusion. ${ }^{3}$ Likewise, the window of time in which NEC is most strongly associated with transfusion is $48 \mathrm{~h}$. Thus, we can surmise that the etiology by which NEC evolves after transfusion unfolds rapidly, although we do not know precisely what the etiology is. Also in this edition, El-Dib et al. demonstrate a reduction in transfusionassociated NEC when feeds were withheld during transfusion. ${ }^{4}$ Finally, no clinical study done thus far has found any relationship between blood product age and NEC (making poor blood banking an unlikely culprit).

The current findings are provocative, but it is first and foremost important to keep equipoise. Retrospective cohort studies cannot prove causation, only association. Thus, the current studies can best be compiled and used as roadmaps for future prospective multicenter studies. The questions we need to ask now are:

(1) What is the risk benefit ratio of transfusion versus anemia with respect to NEC? (2) What is the best way to manage feeding during and after transfusion? (3) Are there variables with blood product preparation that we have yet to properly evaluate (for example, specific blood types, sex, race, seasonality, or preservative use)?

(4) Are there other confounding variables that we have yet to identify besides formula versus breast milk feeds?

There is a lot to do, but suddenly it feels like we have a reason to get on with it. It feels like we could someday conquer NEC ... one subgroup at a time.

\section{Conflict of interest}

The author declares no conflict of interest.

\author{
PV Gordon \\ Department of Pediatrics, Tulane University School \\ of Medicine, New Orleans, LA, USA \\ E-mail:pgordon1@tulane.edu
}

\section{References}

1 Gordon PV, Swanson JR, Attridge JT, Clark R. Emerging trends in acquired neonatal intestinal disease: is it time to abandon Bell's criteria? J Perinatol 2007; 27(11): 661-671.

2 Christensen RD, Lambert DK, Henry E, Wiedmeier SE, Snow GL, Baer VL et al. Is 'transfusion-associated necrotizing enterocolitis' an authentic pathogenic entity? Transfusion 2010; 50(5): 1106-1112.

3 Singh R, Visintainer PF, Frantz ID, Shah BL, Meyer KM, Favila SA et al. Association of necrotizing enterocolitis with anemia and packed red blood cell transfusions in preterm infants. J Perinatol 2011; 31: 176-182.

4 El-Dib M, Narang S, Lee E, Massaro AN, Aly H. Red Blood Cell Transfusion, Feeding and Necrotizing Enterocolitis in Preterm Infants. J Perinatol 2011; 31: 183-187. 